ENTANGLED EMPIRES 
This page intentionally left blank 


\title{
ENTANGLED EMPIRES
}

\section{THE ANGLO-IBERIAN ATLANTIC, \\ I500-I830}

EDITED BY

JORGE CAÑIZARES-ESGUERRA

\author{
$\overline{\text { PENN }}$ \\ UNIVERSITY OF PENNSYLVANIA PRESS \\ PHILADELPHIA
}


Copyright (C) 2018 University of Pennsylvania Press

All rights reserved. Except for brief quotations used for purposes of review or scholarly citation, none of this book may be reproduced in any form by any means without written permission from the publisher.

Published by

University of Pennsylvania Press

Philadelphia, Pennsylvania I9IO4-4II2

www.upenn.edu/pennpress

Printed in the United States of America on acid-free paper IO $98 \begin{array}{llllllll} & 8 & 6 & 5 & 4 & 3 & 2 & \text { I }\end{array}$

Library of Congress Cataloging-in-Publication Data Names: Cañizares-Esguerra, Jorge, editor.

Title: Entangled empires : the Anglo-Iberian Atlantic, I500-I830 / edited by Jorge Cañizares-Esguerra.

Other titles: Entangled empires (2018)

Description: Ist edition. | Philadelphia : University of Pennsylvania Press, [2018] | Includes bibliographical references and index.

Identifiers: LCCN 2017027I09 | ISBN 9780812249835

(hardcover : alk. paper)

Subjects: LCSH: Atlantic Ocean Region-History. | Iberian Peninsula-History. | Great Britain-Foreign relationsSpain. | Spain-Foreign relations_-Great Britain. |

Great Britain-Foreign relations_-Portugal. | Portugal_Foreign relations-Great Britain. | Civilization, Modern. | History, Modern.

Classification: LCC D210.E57 2018 | DDC $303.48 / 218210903-\mathrm{dc2} 3$

LC record available at https://lccn.loc.gov/2017027I09 
To Christopher Schmidt-Nowara and his generous, transoceanic life 
This page intentionally left blank 